\title{
KELEA, Cosmic Rays, Cloud Formation and Electromagnetic Radiation: Electropollution as a Possible Explanation for Climate Change
}

\author{
W. John Martin \\ Institute of Progressive Medicine, South Pasadena, CA, USA \\ Email: wjohnmartin@ccid.org
}

Received 11 January 2016; accepted 27 February 2016; published 1 March 2016

Copyright (C) 2016 by author and Scientific Research Publishing Inc.

This work is licensed under the Creative Commons Attribution-NonCommercial International License (CC

BY-NC).

http://creativecommons.org/licenses/by-nc/4.0/

(c) () (9) Open Access

\section{Abstract}

The basic premise of this article is that human generated electromagnetic radiation is contributing to global warming. It may do so by diverting an energy force termed KELEA (kinetic energy limiting electrostatic attraction) from its presumed association with cosmic rays. Cosmic ray delivered KELEA is viewed as normally participating in the formation of cloud condensation nuclei (CCN). It may do so by transforming electrostatically inert particles into electrostatic aerosols capable of acting as CCN. The resulting clouds act as a reflective barrier to some of the infrared radiation from the sun and, thereby, reduce the earth's heat. This article proposes that increasing levels of electromagnetic radiation in the atmosphere is reducing the capacity of cosmic rays to deliver adequate KELEA to maintain climate stability through optimal cloud formation. Specifically, the fluctuating electrical fields accompanying electromagnetic radiation may do so by competitively withdrawing some of the KELEA from the incoming cosmic rays. Previously described studies by Dr. Wilhelm Reich attributed to an energy force termed orgone, are consistent with weather activity being inducible using a device that likely delivers KELEA to the atmosphere. In addition to the foregoing consideration, there are many agricultural and industrial applications of KELEA activated fluids that can reduce carbon emissions. It is important that the scope of climate science be broadened to include a detailed understanding of KELEA and of its many potential practical applications in addressing global warming.

\section{Keywords}

Climate Change, Cloud, Cosmic Rays, Electromagnetic Radiation, KELEA, Orgone, Water, Cell Phone 


\section{Introduction}

The reality of climate change is finally being acknowledged by world leaders. While of little comfort to those already subjected to disastrous weather conditions, there is optimism that efforts to reduce industrial carbon emissions will lead to more stability in the world's weather system. Climatologists are unlikely to be aware of recent research pointing to a natural force termed KELEA (kinetic energy limiting electrostatic attraction). KELEA is considered a fundamental force of Nature [1]. Its primary purpose may be to prevent the fusion and possible annihilation of opposite electrical charges. KELEA is presumably attracted to separated electrical charges in a manner that provides a barrier to actual fusion of opposite charges as they approach one another due to increasing electrostatic attraction. KELEA may similarly provide the repulsive force between like charges. A further proposal is that KELEA may operate to weaken the strength of intermolecular hydrogen bonding between molecules [2]. This effect has been most clearly documented with water, but also can be seen with other liquids, including gasoline [2]. It may also influence intermolecular electrostatic attraction in gaseous fluids, such as propane and natural gas.

\section{KELEA Activation of Water}

Various dipolar compounds with separated electrical charges can directly attract KELEA from the environment. Certain of these compounds can transfer KELEA to nearby water, possibly in an oscillatory manner [3]. Water can also be activated by being placed within energy fields, typically created by the repetitive on-off switching of electrical devices [3]-[5]. The attracted and subsequently released concentrated levels of KELEA are apparently sufficient to directly activate nearby regular water.

KELEA activated water is characterized by having lowered surface tension and reduced freezing and boiling points and by becoming increasingly volatile (measurable as weight loss in closed but not completely sealed containers) [4]-[6]. At a sufficient level of activation, the separated electrical charges on the water molecules can directly absorb KELEA leading to further activation of the water and to the activation of added water. This principle explains the retention of biological activity in dilute homeopathic remedies [7] [8]. It also allows for the subsequent removal of the water activating compounds from the activated water by decanting or by zero residue filtration [7].

The continuing extraction of KELEA by activated fluids can occur over periods of several months. This was shown by the progressively increasing vapor pressure of activated fluids stored within initially flaccid, sealed plastic containers [2]. In performing these and similar experiments, it was clearly apparent that KELEA attraction was not restricted to daylight hours. Yet, there were notable fluctuations in KELEA over time, which could not be correlated with time of day or night, temperature or the phase of the moon [2]. While not excluding the importance of local influences, such as the nearby presence of dipolar compounds or previously activated fluids, it was concluded that KELEA is variably, but constantly present as a natural environmental force.

\section{KELEA, Cosmic Rays and Cloud Formation}

Since KELEA is a feature that accompanies separated electrical charges and cosmic rays are electrical charged particles, it is reasonable to assume that KELEA is continually being delivered to the earth in association with cosmic rays. Interestingly, if KELEA is the repulsive force between like electrical charges, it could assist in maintaining the flow of cosmic rays within the universe. KELEA may also explain some of the phenomena currently attributed to dark energy, such as the expanding universe [9].

Drs. Sevenmark, Shaviv and others [10]-[15] have proposed that cosmic rays promote low level cloud formation in a manner that the clouds then act as reflecting shields repelling some of the sun's infrared (heating) radiation. According to these investigators, electrical particles emitted by the sun (solar wind) periodically divert some cosmic rays from directly entering the earth's atmosphere and may, thereby, reduce cloud formation. It has proven difficult, however, to directly correlate fluctuating levels of cosmic rays and solar activity with changes in the earth's temperature [16].

Clouds form from water condensing on electrically charged aerosol particles termed cloud condensation nuclei (CCN). Others have presumed that the charged cosmic rays transferred their electrical charges to uncharged, inert particles in the atmosphere and, thereby, create CCN. As an alternative explanation, the inert particles may actually have internal electrical charges that are somewhat masked by electrostatic attraction. In this state they 
do not act as effective CCN. They may, nevertheless, be sufficiently charged to attract the KELEA accompanying cosmic rays, leading to more effective charge separation and CCN formation. Similarly, electrical charges on water molecules may become better exposed by KELEA adding to the greater consolidation of water onto the KELEA generated CCN.

\section{KELEA Diversion to Electromagnetic Radiation}

The basic premise of this paper is that some of the KELEA presumably being delivered to the earth via cosmic rays is being increasingly diverted away from the formation of CCN by its binding to electromagnetic transmissions; resulting in reduced formation of earth cooling, infrared-reflecting clouds. Although this premise can not presently be directly tested, it is consistent with several experimental observations. First, KELEA is presumably attracted to the vicinity of converging electromagnetic fields established using sets of opposing pairs of light emitting devices [4]. This conclusion stems from apparent KELEA-related health benefits occurring in individuals spending several hours in "energy" rooms equipped with two of more pairs of facing computers. The computer screens, which are continually displaying complex changing patterns, are placed so that their light emissions are directed to a centralized area within the room (www.eesystems.com). The attraction and subsequent release of KELEA (scalar energy) is predictably occurring because of fluctuating propulsion towards one another of opposite electrical charges from the electrical fields of the converging light paths. Accordingly, a similar effect can be achieved by using four diagonally placed, inwardly facing, LED traffic lights with an overhead strobe light [4]. A heightened level of KELEA in the therapy room is supported by a reduction from 72 dynes/sq. $\mathrm{cm}$ to $<50$ dynes/sq. $\mathrm{cm}$ in the surface tension of water placed into the therapy room. (The reduction in surface tension is taken as a surrogate marker for water activation). Water placed within the area illuminated by the traffic lights similarly becomes activated, as shown by its increased volatility [4] and by its lower surface tension.

Another important observation is that when the opposing computers within the scalar energy room are operating, the normally present $60 \mathrm{~Hz}$ electromagnetic field (EMF) within the room is no longer detectable using an EmdexII/1400 EMF meter. Moreover, a considerable reduction also occurs in the ambient radio frequencies as measured using a Wandel \& Geltermann EMR-30 radio frequency meter (unpublished).

It is reasonable, therefore, to propose that KELEA can be competitively transferred between different sources of available electromagnetic radiation. The worldwide transmission of electromagnetic energies is continuing to increase with technological advances in communications and with more extensive transmissions of electrical power [17]. This has been especially marked in the Northern Hemisphere, which has warmed in excess of the Southern Hemisphere [18]. Some of the KELEA that might normally participate in cloud formation may instead be diverted to the increasing atmospheric levels of mankind generated electromagnetic radiation. Global warming may, therefore, comprise an added feature of what is commonly called "electropollution."

\section{Electropollution}

The primary focus of potential adverse effects of electromagnetic radiation has been on human health. Of particular concern is the possibility of brain damage resulting from the use of cell phones [19]. The mechanism of damage has been somewhat over simplified as resulting from the local induction of heat by the microwaves relayed by the cell phones. This suggestion of temperature related damage is not well supported by experimental data. Nor does it explain the unique sensitivity displayed by certain individuals to the use of cell phones. It is noteworthy that upon questioning, many of the affected individuals have considerable difficulties in coherently describing their disorder. This cognitive disorder is likely by itself to be a reflection of an underlying brain illness. The author has recently proposed that the fluctuating electrical charges in the brain normally act as an antenna to attract KELEA into the body [20]. The KELEA could function in enhancing the membrane potential of neurons and, thereby, assist in the specificity of various brain functions. Brain attracted KELEA may also provide an added kinetic property to the body's fluids; improving many biological functions. Mental illnesses are reasonably viewed as a deficiency of KELEA. The adverse effect of using a cell phone may be explained by the further withdrawal of KELEA from the brain. The concept that microwaves can extract KELEA from activated water is consistent with the recommended avoidance of exposing homeopathic remedies to microwaves. Similar considerations of possible withdrawal of KELEA can apply to reported adverse effects of other sources of electromagnetic radiation on human and animal health and on agriculture. 


\section{Evidence for the Two-Way Transfer of KELEA}

A yet to be explained phenomenon in performing some of the water activation tests has been the repeated documentation of minor increases in the weight of certain water containing vials compared to the prior measurements of the same vial. The increases are clearly not due to differences in the settings of the weighing balance, since the changes are seen with only certain vials within a given experiment. A larger study has been completed confirming that aluminum foil can also show significant, although minor, periodic fluctuations in weight. These changes are consistent with the addition and release of KELEA leading respectively to an increase and a decrease in measured weight.

This observation raises two possibilities. First, in addition to increased volatility of KELEA activated water, the release of KELEA may lead to a slight loss in the weight manifestation of gravity. The second conclusion is that KELEA activation is presumably reversible by means other than the selective dissipation by evaporation of the more kinetically active water molecules. It was previously suggested that the base of tall trees may be able to remove KELEA from activated water as a necessary requirement for the cohesive transport of water to the upper branches of the tree [7]. While further studies are clearly needed, these considerations are consistent with KELEA being a removable quality from various carriers. They are also consistent with the basic premise of this article that KELEA may be diverted from cloud formation by becoming attached to the electrical charges accompanying electromagnetic radiation.

\section{KELEA and Orgone Energy}

It is useful to consider the possible relationship between KELEA and some of the earlier described energies that have been largely discounted by mainstream science. These forces include the radiant or impulse energy proposed by Nikola Tesla [21]. It is also consistent with the odic energy identified by Karl von Reichenbach [22] and the orgone energy described by Wilhelm Reich [23]. Although not formally presented as scientific literature, Dr. Reich's studies and subsequently described observations by Mr. Trevor Constable [24] and Dr. James DeMeo [23] are particularly germane to the issue of KELEA and climate change. They were each able to induce rain using devices, inappropriately termed cloud busters, which seemingly transmit a non-conventional energy termed orgone. The initial devices comprised no more than upwardly pointing hollow metal tubes with one end placed in flowing water. Conceivably, the orgone devices projected KELEA from the activated water to the atmosphere, where it facilitated the condensation of water vapor into water droplets. This presumably occurred through the formation of CCN. Projected orgone energy was also stated to dissipate smog, potentially by allowing for the formation of larger aerosol particles that sediment. Unfortunately, detailed results from some of the studies relating to orgone were suppressed, while others have been rendered somewhat questionable under the cover of being proprietary. Still, the descriptions of successful manipulation of weather patterns are sufficiently compelling that they cannot be left unaddressed.

\section{KELEA Activated Fluids Can Reduce Carbon Emission}

An attractive aspect of linking global cooling with KELEA is that an understanding of the science of fluid activation can provide many additional opportunities to reduce global warming beyond the potential of providing the earth with more efficient cloud cover. Specifically, utilizing KELEA activated fluids can immediately address the more widely accepted, although still not formally proven argument that global warming is primarily due to the increased burning of fossil fuels. Published studies have shown that less energy to required to heat KELEA activated water. This alone can provide substantial savings in the operations of power plants and in heating, ventilation and air conditioning (HVAC) systems [6]. There is also less corrosion and scale formation in metal pipes carrying activated water [6]. Concrete prepared in activated water is less brittle with a higher tensile strength leading to reduced quantities required in construction (unpublished). Many other industrial applications are under consideration, not only with regards to water but also as applied to gasoline, diesel and gases. Activated gasoline burns more completely, with less hydrocarbon and carbon monoxide emission and at a reduced temperature.

There are also potential enormous benefits of activating water in agriculture and animal husbandry. Using simple means of KELEA activation of water led to increased productivity and longer shelf life of harvested rice, sugarcane and other crops [25]. It also increased the resistance to infectious diseases and rodent infestation of 
the crops [25]. There is likely to be more efficient uptake of water by the treated crop fields, such that the overall demand for agricultural water can be decreased, as can the use of fertilizers. Presently unusable contaminated water can be easily cleansed through the KELEA activation process for reuse (unpublished). The many potential human health benefits of consuming KELEA activated water have also been discussed [7] [26] [27]. It will be of interest to match the relative benefits in reducing global warming by providing more cloud cover versus reducing carbon emission. Certainly both approaches should be actively pursued.

\section{Summary}

This article outlines a possible scenario in which KELEA (kinetic energy limiting electrostatic attraction) brought to the earth by cosmic rays, participates in the formation of heat-reflective cloud cover by activating cloud condensation nuclei (CCN). This process may be rendered less effective if some of the KELEA is removed from cosmic rays by its attachment to fluctuating electrical fields that accompany the increasing electromagnetic radiations present within the earth's atmosphere. The proposed reduction in cloud formation may potentially be remediated by devising alternative means of delivering KELEA to the atmosphere. Moreover, an understanding of KELEA can immediately lead to significant worldwide reductions in carbon emissions.

\section{Acknowledgements}

The Institute of Progressive Medicine is a component of MI Hope Inc., a non-profit public charity.

\section{Conflict of Interest}

None.

\section{References}

[1] Martin, W.J. (2014) Stealth Adapted Viruses; Alternative Cellular Energy (ACE) \& KELEA Activated Water. Author House IN, p.321.

[2] Martin, W.J. (2015) KELEA: A Natural Energy that Seemingly Reduces Intermolecular Hydrogen Bonding in Water and Other Liquids. Open Journal of Biophysics, 5, 69-79. http://dx.doi.org/10.4236/ojbiphy.2015.53006

[3] Martin, W.J. (2015) Methods of KELEA Activation of Water and Benefits in Health, Agriculture and Industry. Journal of Water Resource and Protection, 7, 1331-1344. http://dx.doi.org/10.4236/jwarp.2015.716108

[4] Martin, W.J. (2015) Interacting Light Paths Attract KELEA (Kinetic Energy Limiting Electrostatic Attraction) and Can Lead to the Activation of Water. Open Journal of Biophysics, 5, 115-121. http://dx.doi.org/10.4236/ojbiphy.2015.54010

[5] Martin, W.J. (2015) Interactive Electric Fields Can Attract KELEA (Kinetic Energy Limiting Electrostatic Attraction) and Can Lead to the Activation of Water. International Journal of Complementary \& Alternative Medicine, 1, Article ID: 00034 .

[6] Martin, W.J. (2015) Improved Efficiency of Heat Exchange Using KELEA Activated Water. Open Journal of Energy Efficiency, 4, 36-43.

[7] Martin, W.J. (2015) Alternative Cellular Energy Pathway Therapy Using KELEA Activated Water. International Journal of Complementary \& Alternative Medicine, 2, Article ID: 00051.

[8] Martin, W.J. and Laurent, D. (2015) Homeopathy as a Misnomer for Activation of the Alternative Cellular Energy Pathway: Evidence for the Therapeutic Benefits of Enercel in a Diverse Range of Clinical Illnesses. International Journal of Complementary \& Alternative Medicine, 2, Article ID: 00045.

[9] Villata, M. (2013) On the Nature of Dark Energy: The Lattice Universe. Astrophysics and Space Science, 345, 1-9. http://dx.doi.org/10.1007/s10509-013-1388-3

[10] Svensmark, H. (1998) Influence of Cosmic Rays on Earth’s Climate. Physical Review Letters, 81, 5027-5030. http://dx.doi.org/10.1103/PhysRevLett.81.5027

[11] Svensmark, H. (2000) Cosmic Rays and Earth’s Climate. Space Science Reviews, 93, 175-185. http://dx.doi.org/10.1023/A:1026592411634

[12] Shaviv, N.J. (2005) On Climate Response to Changes in the Cosmic Ray Flux and Radiative Budget. Journal of Geophysical Research: Space Physics, 110, A08105. http://dx.doi.org/10.1029/2004ja010866

[13] Ney, E.R. (1959) Cosmic Radiation and Weather. Nature, 183, 451-452. http://dx.doi.org/10.1038/183451a0 
[14] Usoskin, I.G. and Kovaltsov, G.A. (2008) Cosmic Rays and Climate of the Earth: Possible Connection. Comptes Rendus Geoscience, 340, 441-450. http://dx.doi.org/10.1016/j.crte.2007.11.001

[15] Pierce, J.R. and Adams, P.J. (2009) Can Cosmic Rays Affect Cloud Condensation Nuclei by Altering New Particle Formation Rates? Geophysical Research Letters, 36, L09820. http://dx.doi.org/10.1029/2009GL037946

[16] Tsonis, A.A., Deyle, E.R., May, R.M., Sugihara, G., Swanson, K., Verbeten, J.D. and Wang, G. (2015) Dynamical Evidence for Causality between Galactic Cosmic Rays and Interannual Variation in Global Temperature. Proceedings of the National Academy of Sciences of the United States of America, 112, 3253-3256. http://dx.doi.org/10.1073/pnas.1420291112

[17] Global Electricity Transmission Report and Database 2016-2025. Global Transmission Reports, 3rd Edition, New Delhi.

[18] Friedman, A.R., Hwang, Y.-T., Chiang, J.C.H. and Frierson, D.M.W. (2013) Interhemispheric Temperature Asymmetry Over the Twentieth Century and in Future Projections. Journal of Climate, 26, 5419-5433. http://dx.doi.org/10.1175/JCLI-D-12-00525.1

[19] Bak, M. and Zmyślony, M. (2010) Effects of Electromagnetic Field from Cellular Phones on Selected Central Nervous System Functions: A Literature Review. Medycyna Pracy, 61, 671-683.

[20] Martin, W.J. (2015) Is the Brain an Activator of the Alternative Cellular Energy (ACE) Pathway? International Journal of Complementary \& Alternative Medicine, 1, 00002. http://dx.doi.org/10.15406/ijcam.2015.01.00002

[21] Tesla, N. (1901) Method of Utilizing Radiant Energy. US Patent No. 685,958.

[22] Reichenbach, K. (1854) The Mysterious Odic Force. Aquarian Press, Wellingborough, 83.

[23] Reich, W. Orgone Biophysical Research Laboratory. www.orgonelab.org

[24] Brown, T.J. (1994) Loom of the Future: The Weather Engineering Work of Trevor James Constable. Borderland Sciences, Eureka, CA, 147.

[25] Martin, W.J. (2014) KELEA Activated Water Leading to Improved Quantity \& Quality of Agricultural Crops. Advances in Plants \& Agriculture Research, 2, 00033.

[26] Martin, W.J. (2015) Alternative Cellular Energy. A Unifying Concept in Complementary Alternative Medicine. International Journal of Complementary \& Alternative Medicine, 1, 00022.

[27] Martin, W.J. (2015) Therapeutic Potential of KELEA Activated Water. International Journal of Complementary \& Alternative Medicine, 1, 00001. http://dx.doi.org/10.15406/ijcam.2015.01.00001

\section{Abbreviations}

KELEA—kinetic energy limiting electrostatic attraction; CCN—cloud condensation nuclei. 